\title{
Saúde Mental e Desempenho Acadêmico: um Estudo com Estudantes de Psicologia
}

\author{
Kaio Novaes Fonseca Melo ${ }^{1}$ (D) , Cattiúscia Batista Bromochenkel ${ }^{2}$ (D)
}

Universidade Estadual do Sudoeste da Bahia-UESB, Jequié, BA, Brasil

\begin{abstract}
Resumo: Em vista da relevância do período e processo de adaptação acadêmica e a importância de estudos neste contexto, a presente pesquisa teve como objetivo analisar a saúde mental de estudantes do curso de psicologia, correlacionando os dados obtidos ao desempenho acadêmico dos alunos avaliados, a partir do histórico acadêmico destes. Realizou-se, então, um estudo, com 52 discentes do curso de psicologia de uma instituição privada de um município no interior da Bahia, descritivo, utilizando abordagem qualiquantitativa, por entender que possibilita uma melhor compreensão do fenômeno. Para as análises dos dados estatísticos utilizou-se o Software de Estatística GNU PSPP ver. 1.2.0, aplicando os testes t-Student para amostras independentes e $U$ de Mann Whitney não paramétrico. Assim, verificou-se que os universitários possuírem ou não algum tipo de adoecimento psíquico não afetará no seu desempenho acadêmico, apesar dos alunos terem vinculado o desenvolvimento de sofrimento mental à graduação. Além disso, a maioria da amostra foi constituída por mulheres, prevalecendo diagnósticos de transtornos ansiosos e depressivos. Os psicofármacos mais utilizados foram aqueles indicados para tais transtornos.
\end{abstract}

Palavras-chave: saúde mental, desempenho acadêmico, universitários

\section{Mental Health and Academic Performance: A Study With Psychology Students}

\begin{abstract}
In view of the relevance of the period and academic adaptation process and the importance of studies in this context, the present research aimed to analyze the mental health of psychology students, correlating the data obtained to the academic performance of the evaluated students, based on the academic background of these. A descriptive study was then carried out with 52 students from the psychology course of a private institution in a municipality in Bahia Countryside, using a qualitative-quantitative approach, as it was understood to enable a better understanding of the phenomenon. For the analysis of statistical data the Statistical Software GNU PSPP ver. 1.2.0, applying Student's t-test for independent samples and nonparametric Mann Whitney $U$ test. Thus, it was found that university students, whether or not they have some kind of mental illness, will not affect their academic performance, although students have linked the development of mental suffering to graduation. In addition, the majority of the sample consisted of women, with diagnoses of anxiety and depressive disorders prevailing. The most used psychotropic drugs were those indicated for such disorders.
\end{abstract}

Keywords: mental health, academic performance, college students

1 Graduando da Faculdade de Tecnologia e Ciências (UniFTC), departamento de Psicologia. Jequié, Bahia, Brasil. E-mail: kaionovaes2@gmail.com

2 Mestranda em Ciências da Saúde pelo Programa de Pós-Graduação em Enfermagem e Saúde, Departamento de Saúde, Universidade Estadual do Sudoeste da Bahia (UESB). Jequié, Bahia, Brasil. E-mail: cattiuscia@gmail.com

Submetido em: 11/03/2021. Primeira decisão editorial: 13/05/2021. Aceito em: 24/06/2021. 


\section{Introdução}

O conceito de saúde mental vai muito além do que apenas a existência ou não de algum transtorno psíquico, perpassando por aspectos comunitários, subjetivos, socioeconômicos, biológicos e ambientais. Na atualidade, a saúde mental pode ser definida como um completo bemestar físico, mental e social, e não apenas a não incidência de adoecimento psicológico (WHO, 2018). Entende-se, então, que o equilíbrio entre bem-estar e saúde mental pode ser comprometido também por variáveis externas, tais como pressões ambientais, mudanças repentinas na rotina, entre outras coisas que estão presentes durante as vivências acadêmicas de estudantes no ensino superior.

Nesta perspectiva, Silva et al. (2019) afirmam que durante a jornada universitária os jovens discentes passam por diversas situações que podem ser avaliadas como estressoras, dentre estas, podem ser citadas o período de provas, as tarefas para além da sala de aula e ainda, o compromisso pessoal com o desempenho. No que diz respeito ao curso de psicologia, soma-se ainda a tais questões as responsabilidades para com o cuidado do outro, tornando o jovem estudante interlocutor das angústias e sofrimentos dos pacientes/usuários do serviço, além de ter que lidar com aspectos éticos e conflitos morais durante as experiências em campo.

Tais situações podem levar à redução do tempo livre para realização de atividades prazerosas como, por exemplo, passar períodos junto a família e amigos e, até mesmo, a realização de algumas necessidades pessoais. Além disso, Cestari et al. (2017) afirma que, apesar dos universitários não terem as responsabilidades de profissionais, estes precisam se adaptar a um novo modelo de vida, assumindo responsabilidades, como estudar, cumprir com avaliações, participar de aulas práticas e estágios. Todas essas circunstâncias favorecem uma condição de maior vulnerabilidade, podendo originar crises situacionais (Nogueira, 2017); tal fato leva a reflexão de que esta população pode ser vista como um possível grupo de risco para o desenvolvimento de sofrimento psíquico.
Corroborando com esta ideia, Costa e Nebel (2018) afirmam que em todo o mundo nota-se que as taxas de doenças mentais como depressão, crise de pânico, distúrbios do sono e risco de suicídio, são maiores entre acadêmicos da graduação. Portanto, a forma como os estudantes subjetivam e introjetam as experiências vividas durante os anos de formação podem levar ao desenvolvimento de sofrimento mental. Assim sendo, pode-se afirmar, então, que a jornada universitária é, ao menos potencialmente, uma experiência estressora para estes alunos.

Contribuem, ainda, a mudança de realidade que os estudantes sofrem ao saírem do ensino médio, insegurança quanto a atuação profissional posterior à formação e a exigência cada vez maior do mercado de trabalho. Neste sentido, literaturas e pesquisadores da área apontam que os discentes do ensino superior estão mais propensos a problemáticas psíquicas que outros grupos populacionais.

É possível citar, também, como dificuldade, a necessidade que muitas vezes esses jovens têm de sair da casa dos pais ao ingressarem no ensino superior, afastando-se de pessoas com quem convive e gosta, como os amigos, círculos seguros e familiares de relacionamentos. Frequentemente, estes representam uma importante rede de apoio. Desta maneira, o distanciamento pode desencadear situações de crises (Almeida et al., 2018).

Portanto, considerando a relevância do período e processo de adaptação acadêmica e a importância de estudos neste contexto, surgiu o seguinte questionamento: como alguns aspectos relacionados à saúde mental podem impactar no desempenho acadêmico de estudantes de psicologia? Nesta perspectiva, o presente estudo teve como objetivo geral analisar a saúde mental de estudantes do curso de Psicologia, a partir de um questionário de saúde mental e, como objetivo específico, correlacionar os dados obtidos ao desempenho acadêmico dos alunos avaliados, utilizando-se o histórico acadêmico destes. Tratase de um estudo epidemiológico, descritivo, utilizando abordagem quali-quantitativa, por entender que possibilita uma melhor compreensão do fenômeno. 
Assim, este estudo justifica-se pelo anseio de contribuir para um entendimento de como os fatores de saúde mental podem interferir no desempenho acadêmico de estudantes de graduação em psicologia e como tais fatores influenciam as vivências universitárias dos discentes, e, desta forma, podendo formular possíveis intervenções para atender às necessidades desta população.

\section{Material e Método}

A pesquisa teve como campo de estudo um município do interior da Bahia e foi aprovada pelo Comitê de Ética em Pesquisa da Universidade Estadual do Sudoeste da Bahia (UESB), com parecer favorável de número 3.905.958. A mostra selecionada foi de 52 estudantes, sendo que o tamanho da população geral era de 200 alunos matriculados, exceto os do primeiro semestre, e foram selecionados grau de confiança de $90 \%$ e margem de erro de $10 \%$

Participaram da pesquisa discentes, de ambos os sexos e idades variadas, regularmente matriculados no curso de psicologia da Faculdade de Tecnologia e Ciências (UniFTC) e que aceitaram participar voluntariamente do estudo. Após o assentimento da coordenação do curso, o pesquisador dirigiu-se a cada turma, em horários de aula, fazendo exposições orais acerca da pesquisa, e assim, convidando quem gostaria de se voluntariar como participante do estudo.

Os estudantes voluntários foram informados sobre os objetivos e procedimentos do estudo e, posteriormente, assinaram o Termo de Consentimento Livre e Esclarecido (TCLE), que atendeu às recomendações da Resolução $n$ 으 466/2012, do Conselho Nacional de Saúde.

Como critérios de inclusão, foram considerados estudantes do curso de psicologia, do segundo ao último período, da Faculdade de Tecnologia e Ciências (UniFTC), de idades variadas, que aceitassem participar voluntariamente do estudo assinando o Termo de Consentimento Livre e Esclarecido (TCLE). Já como critérios de exclusão, empregou-se os seguintes parâmetros: discentes que não aceitaram oferecer os dados de desempenho acadêmico, menores de 18 anos, estudantes ingressos no primeiro período letivo (visto que estes ainda não têm histórico acadêmico) e pessoas que não optaram por participar voluntariamente do estudo através da assinatura do TCLE.

A coleta dos dados foi realizada durante o mês de março/2020, por meio de encontros presenciais, em uma das salas da própria universidade. Neste período, foi solicitado aos estudantes a disponibilização do histórico acadêmico por via digital. A média de notas do histórico acadêmico foi a base de dados para avaliação do desempenho acadêmico dos alunos e foi pareado com informações do questionário. Procedeu-se então com a aplicação, de maneira coletiva, um questionário de saúde mental com os discentes, contendo sete assertivas com caráter objetivo.

As perguntas tiveram como objetivo levantar informações quanto a possíveis diagnósticos de transtornos mentais entre os participantes do estudo, além do uso ou não de psicofármacos; se o estudante é acompanhado ou já buscou algum tipo de assistência psiquiátrica e/ou psicológica, se há histórico de transtorno mental na família; houve desenvolvimento de sofrimento psíquico após o ingresso na faculdade - "após entrar na Universidade você passou a ter algum tipo de sofrimento psíquico? Se sim, qual?"”, seguido de uma escala do tipo Likert, de um a cinco, de como o sujeito autoavalia sua saúde mental. Esta, era dividida em escala de péssimo, ruim, regular, bom e excelente, contemplando 5 itens.

Posteriormente, foi realizada a tabulação dos dados obtidos a partir dos questionários em uma planilha criada no Software Microsoft Office Excel ${ }^{\circledR}$ 2003. Por fim, para as análises dos dados estatísticos, utilizou-se o Software de Estatística GNU PSPP ver. 1.2.0, aplicando os testes t-Student para amostras independentes e $U$ de Mann Whitney não paramétrico.

\section{Resultados}

A amostra foi composta por 52 discentes, tendo como maioria pessoas do gênero feminino, representando $86,8 \%$ da população. Já os estudantes do gênero masculino, representaram $13,2 \%$ dos 
participantes. Dentre a população analisada, $10 \%$ relatou ser diagnosticado com algum tipo de transtorno mental, sendo os mais comuns: transtornos ansiosos e depressivos.

Evidenciou-se, ainda, que $48 \%$ dos alunos afirmaram que nunca fizeram acompanhamento psiquiátrico ou psicológico; enquanto que $23 \%$ disseram já haver feito ou ainda continuam atualmente o tratamento, este iniciado anterior ao ingresso no universo acadêmico. Por fim, $29 \%$ da amostra declarou o começo somente após a entrada na faculdade.

Quanto à farmacoterapia, $6 \%$ dos estudantes afirmaram utilizar algum tipo de medicamento atualmente, prevalecendo medicamentos indicados para o tratamento dos transtornos anteriormente citados, tais como Rivotril ${ }^{\circledR}$, Alprazolam, Sertralina, Trazodona e Mirtazapina.

Quando questionados se houve desenvolvimento de algum tipo de sofrimento psíquico após o ingresso no ensino superior, 52\% da amostra declarou que não, enquanto $46 \%$ afirmou que houve desenvolvimento de sofrimento psicológico e $2 \%$ dos participantes não responderam à pergunta; ao solicitar a identificação destes sofrimentos, 31\% dos alunos explicitaram que, após o ingresso na universidade, passaram a sentir-se mais ansiosos.

Disseram ainda de que, ao final do semestre, com o período de provas, encurtamento de prazos e cobranças dos professores, experenciam agitação psicomotora e mudanças no padrão de sono-vigília. Tais dados foram alcançados através da pergunta: "após entrar na Universidade você passou a ter algum tipo de sofrimento psíquico? Se sim, qual?", no qual os participantes puderam relatar os sinais e sintomas que associavam a seu sofrimento psíquico.

A comparação estatística entre as médias dos discentes que afirmaram ser diagnosticados com algum tipo de transtorno mental e aqueles que disseram não haver nenhuma descrição diagnóstica, podem ser observadas nas tabelas 1 e 2, a seguir:

\section{Tabela 1}

Médias do desempenho acadêmico de alunos do curso de Psicologia de uma Faculdade no Estado da Bahia.

\begin{tabular}{l|r|r}
\hline \multicolumn{1}{c|}{ Condição Mental } & $\begin{array}{r}\text { Desempenho } \\
\text { acadêmico }\end{array}$ & \multirow{2}{*}{ Teste t } \\
\hline $\begin{array}{l}\text { Presença de } \\
\text { Transtorno }\end{array}$ & 77,96 & \\
\hline Ausência de & 70,88 & \\
Transtorno & $1,78^{\text {ns }}$ \\
\hline
\end{tabular}

\section{Tabela 2}

Correlação entre as médias de acadêmicos que afirmaram ter algum transtorno mental (TM) diagnosticado e os que disseram não haver.

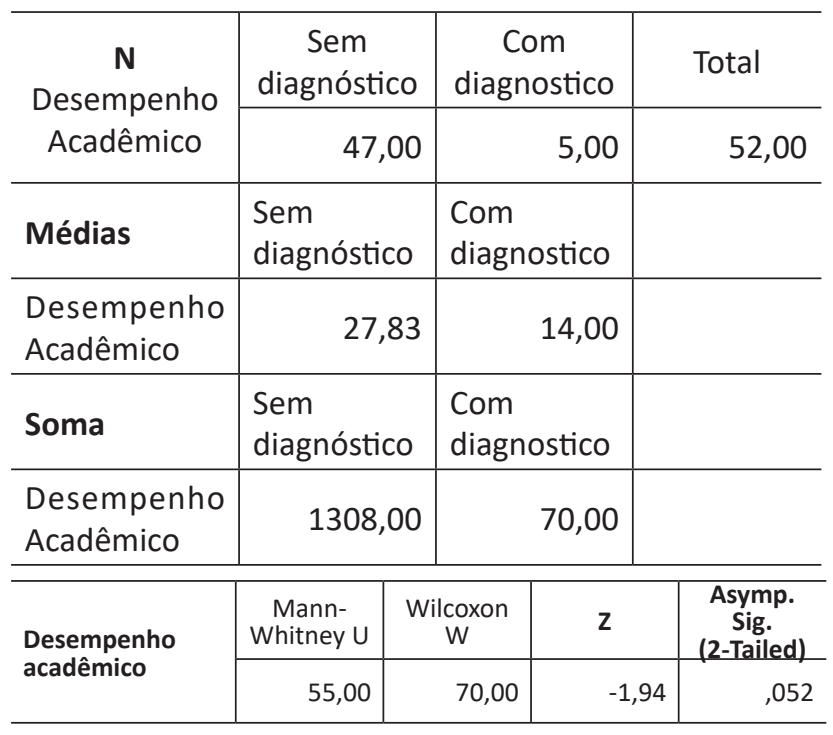

Como observado anteriormente, a partir do teste t-Student para amostras independentes, relacionou-se as médias dos alunos que afirmaram possuir algum tipo de diagnóstico de transtorno mental com aqueles que declararam não haver descrição diagnóstica. Sendo o valor de $t$ calculado igual a 1,78 e o valor de $t$ tabelado igual a 2,02 . Portanto, o valor de $t$ calculado é menor que o valor de $t$ tabelado. Desta forma, foi possível concluir que não há diferenças significativas entre as médias das duas amostras ao nível de significância de 5\%.

Tal conclusão também foi corroborada pelos resultados obtidos pelo teste $U$ de Mann Whitney, reafirmando tal achado. Neste foi delimitado, como hipótese nula ( $\mathrm{HO})$, que as médias entre os alunos com transtorno diagnosticado e alunos sem transtorno diagnosticado são iguais estatisticamente e, como hipótese alternativa ( $\mathrm{H} 1)$, que as médias entre estes 
dois grupos diferem estatisticamente. Foi achado o P-valor 0,052 maior que o nível de significância (nível alfa $5 \%, 0,05)$.

No que diz respeito ao histórico de transtorno mental na família, $19 \%$ da amostra afirmou ter familiares com algum tipo de transtorno psiquiátrico, enquanto que $81 \%$ declarou não haver nenhuma prevalência de distúrbio psíquico em parentes. A respeito da escala do tipo Likert utilizada no questionário, $11 \%$ dos estudantes se autoavaliaram com a saúde mental ruim; $47 \%$ da população estudada avaliou como regular; $38 \%$ como bom e $4 \%$ excelente.

Utilizando o teste $\mathrm{t}$-Student, comparouse estatisticamente as médias dos discentes que afirmaram ser diagnosticados com transtornos mentais com aqueles que disseram não haver nenhuma descrição diagnóstica. Concluiu-se que não há diferenças significativas entre as médias das duas amostras ao nível de significância de $5 \%$. Tal resultado também foi evidenciado com o teste $U$ de Mann Whitney.

\section{Discussão}

\section{Saúde Mental de Estudantes do Curso de Psicologia}

Ao considerar a importância do estudo de como a saúde mental dos estudantes pode ser impactada por fatores acadêmicos, questionou-se quanto à existência de diagnóstico de transtorno mental, sendo possível notar que $10 \%$ dos alunos do curso de psicologia afirmaram ser diagnosticados com algum tipo de transtorno mental, predominando descrições relativas à ansiedade e depressão. Entretanto, não foi possível realizar uma associação clara entre o diagnóstico destas psicopatologias concomitante ao início das vivências acadêmicas, uma vez que tais descrições nosográficas podem estar relacionadas às experiências prévias ao início do ensino superior.

Em um estudo realizado por Barros et al. (2017), foram avaliados, pela escala PHQ-9 (9-item Patient Health Questionnaire), 49.025 adultos com idades que variam entre 18 a 59 anos. Segundo os resultados alcançados, $9,7 \%$ dos adultos brasileiros apresentaram depressão e 3,9\%, depressão maior. Nesta pesquisa, cerca de $21,0 \%$ relataram humor depressivo e, em 34,9\% deles, esse sentimento esteve presente por mais de sete dias; $7,2 \%$ dos entrevistados já receberam, em algum momento da vida, diagnóstico clínico da doença.

Portanto, ao observar as conclusões encontradas na pesquisa anterior, é possível afirmar que não há variação significante entre os informes apresentados por Barros et al. (2017) e o percentil de discentes que afirmaram possuir o diagnóstico de depressão, sugerindo que, neste caso, a universidade não atuou como variável determinante para identificação da depressão.

Referente à ansiedade, observando os relatórios apresentados pela World Health Organization (WHO), em 2017, a respeito das estimativas de transtornos globais de saúde, é perceptível a manifestação do transtorno de ansiedade em cerca de 9,3\% da população brasileira. Tal fato evidencia que a porcentagem de estudantes do curso de psicologia que relataram ter descrições diagnosticas relativas à ansiedade, aproxima-se de números da psicopatologia da população nacional.

Assim sendo, considerando todas informações anteriormente apresentadas, pode-se concluir que a universidade não atuou como variável determinante para o diagnóstico de transtornos ansiosos e depressivos em estudantes de psicologia, apesar de serem encontrados como os principais transtornos referidos na pesquisa.

Outro fato importante diz respeito à prevalência destes transtornos em mulheres; vários estudos da área apontam que a ansiedade e a depressão são mais comuns em pessoas do sexo feminino (Organização Mundial da Saúde [OMS], 2018; Costa et al., 2019). Partindo do entendimento de que, no presente estudo, $86,8 \%$ das participantes são mulheres, tal fato acaba por indicar a relação com gênero também na amostra investigada.

Para além de tais experiências e fatores prévios ao ingresso na faculdade, é importante levar em conta que os anos passados, acontecimentos e episódios enfrentados na academia podem desencadear situações de crise, levando, assim, ao sofrimento mental dos estudantes. Partindo desse 
princípio, foi questionado quanto ao surgimento do sofrimento psíquico posterior ao ingresso na universidade, sendo possível observar que $46 \%$ da amostra afirmou ter notado o aparecimento de algum tipo de padecimento psíquico após o ingresso no ensino superior. Quando questionado sobre a especificidade de tais angustias, foram relatados o desenvolvimento de sentimentos de fracasso, incapacidade e inferioridade; incompetência, insuficiência, tristeza e desespero.

Tais representações mentais e percepções da problemática condizem com as apresentações de sinais e sintomas descritas pelo Manual de Diagnóstico e Estatístico de Transtornos Mentais (DSM-V) e Classificação Internacional de Doenças (CID-10) comuns aos transtornos depressivos, uma vez que houve prevalência de interpretações caracteristicamente negativas de si, do mundo e do futuro, além de humor deprimido, perda de interesse e prazer e energia reduzida. Entretanto, salienta-se que não é possível afirmar diagnósticos, visto que este não foi o objetivo do estudo e características como tempo e intensidade dos sintomas não foram avaliados.

Corroborando com tais achados, os mesmos aspectos foram relatados na literatura como comuns em pessoas que frequentam o ensino superior (Fórum Nacional de Pró-Reitores de Assuntos Comunitários e Estudantis [FONAPRACE], 2018; Carvalho et al., 2015; Padovani, et al., 2014). Entretanto, apesar disso, por não ter sido avaliados os critérios de tempo e intensidade, não é possível que há incidência de transtornos depressivos nos estudantes, mas sim sentimentos e pensamentos que são comuns na depressão.

Contribuindo com a discussão, Dalgalarrondo (2019) descreve os componentes do surgimento, constituição e manifestação dos sintomas e dos transtornos mentais, dividindo-os em fatores predisponentes, relacionados à carga genética, experiências emocionais no início da vida e do período da adolescência, e precipitantes, ligados a eventos da vida, como estresses e situações atuais. Ambos influenciam diretamente no surgimento dos transtornos mentais. Pode-se concluir, então, ser preciso levar em conta a maneira como se relaciona ao longo da história de vida do sujeito, os diversos conjuntos de fatores biológicos, psicológicos e sociais para a emergência ou não de sintomas ou transtornos psíquicos.

Desta forma, é possível identificar como fatores precipitantes na população estudada a universidade e as experiencias associadas a ela, como o período de provas, encurtamento de prazos e cobranças dos professores, descritas pelos alunos. Considerando, ainda, a população do estudo, podem ser definidos como fatores predisponentes o histórico de transtornos mentais na família, afirmado por $19 \%$ dos alunos. Portanto, é possível inferir que estes fatores podem levar a uma vulnerabilidade do ponto de vista psicológico, reforçando, assim, a hipótese de que, para boa parte da amostra investigada, o ensino superior atuou como fator estressor e causador de sofrimento mental.

Assim sendo, de acordo com os dados supracitados, identificou-se que parcela expressiva (46\%) dos estudantes de psicologia apresenta a percepção de desenvolvimento de sofrimento mental posterior ao início das experiências vividas na universidade. Neste sentido, é possível afirmar que, para estes discentes, a faculdade atuou como fator estressor e causador de sofrimento psíquico.

Em relação à busca por assistência psicológica, de acordo com Taborda (2015), boa parte dos discentes apresentam resistência no que diz respeito à procura profissional. Assim sendo, a literatura da área aponta que apenas pequena parte dos estudantes procuram pela assistência especializada. Considerando tal informação, ao analisar os resultados obtidos no presente estudo, foi possível perceber que $48 \%$ dos participantes declararam nunca ter realizado acompanhamento com algum especialista da área de saúde mental. Em contrapartida, $23 \%$ dos estudantes afirmaram já ter feito ou continuam atualmente em tratamento, tendo iniciado antes mesmo do ingresso na faculdade e, por fim, 29\% da amostra declarou a busca por assistência psíquica somente após o ingresso na universidade.

Portanto, é possível concluir que os achados do presente estudo, referente à busca por tratamento psíquico entre os acadêmicos, diferem dos dados divulgados por autores e pesquisas científicas da área, ao abordarem semelhante problema e 
temática, uma vez constatado que mais da metade do percentil da amostra estudada afirmou recorrer ao auxílio de um especialista. Considerando que a percepção do adoecimento mental é um importante elemento para a busca por tratamento psicológico, ao questionar a respeito de como os estudantes autoavaliam a sua saúde mental, $11 \%$ da amostra afirmou definir esta como ruim; $47 \%$ da população estudada avaliou como regular; $38 \%$ como bom e, por fim, $4 \%$ excelente.

É perceptível que a maioria dos alunos se consideram com a saúde mental regular e boa. Tal ocorrência pode estar ligada ao número expressivo de estudantes que procuram por cuidados o profissional psicólogo ou psiquiatra. Ainda a respeito destas informações, correlacionando a realização de tratamento com um profissional, verifica-se que há uma pequena diferença entre os números daqueles discentes que declararam a busca por acompanhamento profissional anterior à faculdade e dos estudantes que procuraram, posteriormente, ao ingresso na universidade.

Este fato pode estar associado ao entendimento da necessidade de tratamento e à aceitação da existência de um problema psíquico, o que representa e perpassa por fatores subjetivos como, por exemplo, a percepção de desenvolvimento de sofrimento psicológico após o início do percurso acadêmico e a autoavaliação da saúde mental.

No que se refere à farmacoterapia, $6 \%$ da amostra afirmou fazer uso de medicamentos atualmente, dentre eles pode-se citar: Rivotril', Alprazolam, Sertralina, Trazodona e Mirtazapina. Os psicofármacos são substâncias químicas sintéticas, capazes de atuar no sistema nervoso central, influenciando desde o humor, percepção e atitudes do sujeito, até mesmo as funções cognitivas. São destinados à terapêutica de psicopatologias e têm como objetivos a remissão, recuperação ou controle dos sintomas (Santos \& Silveira, 2019).

0 Rivotril (clonazepam) pertence à classe dos benzodiazepínicos, medicamentos que causam inibição leve do sistema nervoso, com consequente ação anticonvulsivante, sedativa leve, relaxante muscular e tranquilizante. Segundo a bula, é indicado para transtornos de ansiedade, transtornos de humor e, em alguns casos, síndromes psicóticas. Da mesma maneira, o Alprazolam, faz parte da mesma classe e possui ação similar.

Já o Cloridrato de Sertralina, é um medicamento antidepressivo, da classe dos inibidores seletivos de recaptação de serotonina, enquanto que a Trazodona, é indicada no tratamento da depressão com ou sem episódios de ansiedade, depressão maior e constitui-se um antidepressivo atípico. Por fim, a Mirtazapina trata-se de um antidepressivo de ação dupla, ou seja, noradrenérgica e serotonérgica (Cordiol et al., 2015). Também é destinada à terapia de estados depressivos.

Desta forma, é possível afirmar que, dos psicofármacos utilizados pela amostra atualmente, prevalecem os ansiolíticos e antidepressivos. Esta questão relaciona-se com outros dados levantados no presente estudo, como a prevalência de diagnósticos depressivos e ansiosos em parte dos acadêmicos de psicologia, corroborado pela literatura científica que aponta tais transtornos como frequentes entre os estudantes de graduação (Rambo et al., 2019; Campos, 2016).

Assim sendo, observando as divulgações cientificas da área a respeito da prevalência do uso de antidepressivos e ansiolíticos entre pessoas que frequentam o ensino superior, é possível afirmar que há proximidade entre estes dados e os informes encontrados na presente pesquisa, uma vez que houve o predomínio da utilização dos mesmos psicofármacos.

Em uma pesquisa realizada por Santos e Silveira (2019), que teve como objetivo investigar a utilização de medicamentos psiquiátricos por estudantes de psicologia de uma faculdade do norte do Rio Grande do Sul, constatou-se o uso de algum psicofármaco em 15,1\% dos 265 participantes, destes, 9,1\% relataram utilizar ansiolíticos. 0 estudo ainda possibilitou a constatação de que a grande maioria dos discentes que faziam terapia, não utilizavam nenhum tipo de fármaco psiquiátrico. Assim, os autores chegaram a um entendimento: aqueles que já haviam feito psicoterapia usavam menos medicação.

Entretanto, apesar da proximidade entre os dados expostos anteriormente, percebe-se que $o$ 
número de discentes do curso de psicologia que faz uso de psicofármacos é significativamente menor, quando comparado com os mesmos índices descritos por outros pesquisadores e autores, ao mencionar estudantes do ensino superior. Tal fato pode estar correlacionado com a incidência de acadêmicos que optaram pela busca por assistência psicológica, isto é, aqueles que fazem ou já fizeram terapia tendem a utilizar menos medicamentos psiquiátricos.

Foi possível observar também que os discentes que informaram haver transtorno mental possuem desempenho acadêmico não significante estatisticamente dos demais alunos, ou seja, o universitário possuir ou não algum tipo de adoecimento psíquico, não impacta diretamente no seu desempenho acadêmico.

Assim sendo, apesar de parte dos estudantes afirmarem ter notado, após à entrada na universidade, surgimento de sinais e sintomas relativos a sofrimento mental, e levando em consideração a hipótese de que estes poderiam influenciar no desempenho acadêmico destes alunos, concluiu-se que tais aspectos parecem não impactar nas médias obtidas pelos discentes ao final do semestre. Portanto, a observação de transtornos mentais nesta população não cria restrições ou tampouco é determinante no desenvolvimento de competências e capacidades nas médias obtidas ao final do semestre.

Outro fator relevante, em relação à percepção de sinais e sintomas apresentados pelos estudantes, é o de que estes podem ser notados ou vivenciados, sem necessariamente compor um transtorno ou preencher categoria diagnóstica, ou até mesmo não foram diagnosticados justamente pela não busca por profissionais especializados. Isto é relevante a fim de que sejam considerados por gerarem sofrimento.

\section{Conclusão}

Este trabalho permitiu analisar a existência não significativa de relação entre saúde mental e desempenho acadêmico, apesar de apresentar a vida universitária como fator estressor para parcela considerável da amostra. Concluiu-se que o universitário possuir ou não algum tipo de adoecimentopsíquiconão afetará noseu desempenho acadêmico. Todavia, é importante sinalizar que se faz necessário investigar outros fatores envolvidos nos anos de formação universitária, possibilitando, assim, uma compreensão mais ampliada do contexto de saúde mental dos estudantes do ensino superior.

Foi possível, também, perceber que, aproximadamente, $13 \%$ dos alunos demostraram sentir-se fracassados, incapazes e inferiores; incompetentes, insuficientes, tristes e desesperados; além de afirmar que, ao final do semestre, com o período de provas, encurtamento de prazos e cobranças dos professores, experenciam agitação psicomotora e mudanças no padrão de sono-vigília, vinculando a emergência destes sentimentos à graduação. Tais problemáticas relacionam-se com a necessária adaptação dos estudantes a novas rotinas e tarefas universitárias, uma vez que, com a entrada na universidade, o aluno passa assumir responsabilidades e vivenciar pressões cotidianas que até então não existiam, o que implica em fatores estressores relacionados à vida acadêmica.

Entretanto, para além das questões aqui discutidas, diversas problemáticas devem ser levadas em consideração, como, por exemplo, os aspectos socioeconômicos e como estes podem impactar nas vivências acadêmicas, permitindo um olhar com maior abrangência de outras variáveis envolvidas nos anos de graduação. Tal fato permitiria pensar em projetos assistenciais que tenham o intuito do amparo à saúde mental e às experiências vividas durante o período de formação desta população, podendo, então, interferir neste cenário, proporcionando aos discentes instrumentos e meios de experienciar a jornada acadêmica de maneira menos sofrida.

Portanto, apesar do presente estudo ter feito contribuições significativas para a investigação da saúde mental em estudantes universitários, este apresenta certas limitações e fragilidades que devem ser pontuadas, no intuito de nortear o levantamento que, por ventura, possa ser realizado por outros pesquisadores. Desta forma, é importante considerar que tais dificuldades permitem a formulação e a possibilidade de realização de mais estudos com esta temática, permitindo, assim, maior expansão em pesquisas com este tema. 


\section{Contribuição}

As pesquisadoras declaram não haver conflitos de interesse. Referências

\section{Referências}

Almeida, L. Y., Carrer, M. O., Pillon, S. C. \& Souza, J. (2018). Avaliação do apoio social e estresse em estudantes de enfermagem. Revista da Escola de Enfermagem da Usp, 52(66), 1-8. doi: 10.1590/s1980-220×2017045703405

Alprazolam: comprimidos. (2016). Responsável técnico: Dra. Maria Benedita Pereira. Eurofarma. Bula de remédio.

American Psychiatric Association. (2014). Manual Diagnóstico e Estatístico de Transtornos Mentais: DSM-5. (5rd ed.). Artmed.

Azevedo, R. C. S., Barros, M. B. A., Lima M. G., Lopes, C. S., Malta, D. C., Medina, L.B.P. \& Menezes P. R. (2017). Depression and health behaviors in Brazilian adults - PNS 2013. Revista de Saúde Pública, 51(1), 1-10. doi: 10.1590/s1518-8787.2017051000084

Campos, C. R. F. (2016). Perfil sociodemográfico, clínico e acadêmico de estudantes universitários que passaram por atendimento psiquiátrico no Serviço de Assistência Psicológica e Psiquiátrica ao Estudante da Universidade Estadual de Campinas (SAPPE-Unicamp) entre $2004 e$ 2011 [Dissertação de mestrado, Universidade Estadual de Campinas].

Bertolini, S. M. M. G., Carvalho, E. A., Milani, R. G. \& Matins, M. C. (2015). Índice de ansiedade em universitários ingressantes e concluintes de uma instituição de ensino superior. Ciência, Cuidado e Saúde, 14(3), 1290-1298. doi: 10.4025/cienccuidsaude.v14i3.23594

Barbosa, I. V., Cestari, V. R. F. Florêncio, R.S., Moreira, T. M. M. \& Pessoa, V. L. M. P. (2017). Estresse em estudantes de enfermagem: estudo sobre vulnerabilidades sociodemográficas e acadêmicas. Acta Paulista de Enfermagem, 30(2) 190-196. doi: 10.1590/1982-0194201700029
Cloridrato de Sertralina: comprimidos. (2019). Responsável técnico: Dr. Ronoel Caza de Dio. EMS. Bula de remédio.

Cloridrato de Trazodona: comprimidos. (2019). Responsável técnico: Dr. Ronoel Caza de Dio. EMS. Bula de remédio.

Cordiol, A. V., Gallois, C. B \& Isolan, L. (Orgs.) (2015). Psicofármacos: consulta rápida. (5rd ed.). Artmed.

Branco, J. C. Costa, C. O., Silva, R. A., Souza, L. D. M \& Vieira, I. S. (2019). Prevalência de ansiedade e fatores associados em adultos. Jornal Brasileiro de Psiquiatria, 68(2), 92-100. doi: 10.1590/0047-2085000000232

Costa, E. G., Nebel, L. (2018). O quanto vale a dor? Estudo sobre a saúde mental de estudantes de pós-graduação no Brasil. Polis, 17(50), 207-227. doi: 10.4067/S0718-65682018000200207

Dalgalarrondo, P. (2019). Psicopatologia e semiologia dos transtornos mentais. (3rd ed.). Artmed.

Fórum Nacional de Pró-Reitores de Assuntos Comunitários e Estudantis. (2018). V Pesquisa Nacional de Perfil Socioeconômico e Cultural dos (as) Graduandos (as) das IFES. http://www.andifes.org. $\mathrm{br} / \mathrm{v}$-pesquisa-nacional-de-perfil-socioeconomico-ecultural-dos-asgraduandos-as-das-ifes-2018

Mirtazapina: comprimidos. (2019). Responsável técnico: Cláudia Larissa S. Montanher. Sandoz do Brasil. Bula de remédio.

Nogueira, M. J. C. (2017). Saúde mental em estudantes do ensino superior: fatores protetores e fatores de vulnerabilidade [Tese de Doutorado, Universidade de Lisboa].

Organização Pan-Americana da Saúde/Organização Mundial da Saúde. (2018). Folha informativa Depressão. https://www.paho.org/bra/index. php?option=com_content $\&$ view $=$ article $\& i d=56$ 35:folhainformativa-depressao\&ltemid=1095.

Organização Mundial da Saúde. (1996). Classificação Estatística Internacional de Doenças e Problemas Relacionados à Saúde: CID-10 Décima revisão. (3rd ed.). EDUSP.

Barbosa, L. N. F., Carmem, B. N., Cavalcanti, H. A. F., Lameu, J. N., Maltoni, J., Padovani, R. C. \& Souza, W. F. (2014). Vulnerability and psychological well-being of college student. Revista Brasileira de Terapias Cognitivas, 10(1), 2-10. doi: 10.5935/1808-5687.20140002 
Rambo, R. R. L; Lima, C. R. L. De; Zorzi, M. R. (2019). A utilização de psicofármacos por acadêmicos do curso de Medicina, em uma universidade no Meio Oeste de Santa Catarina, matriculados em 2017. Revista da Amrigs, 63(1), 43-48.

Rivotril: clonazepam. (2017). Responsável técnico Tatiana Tsiomis Díaz Roche. Bula de remédio.

Santos, L. H.; Silveira, M. M. (2019). Uso de psicofármacos por estudantes de psicologia. Cadernos Brasileiros de Saúde Mental, 11(29) 1-12. doi: https://doi.org/10.5007/cbsm. v11i29.69801

Batista, K. M., Costa, A. L. S., Mussi, F. C., Silva, R. M., Lopes, V. C \& Santos, O. P. (2019). Health alterations in nursing students after a year from admission to the undergraduate course. Revista da Escola de Enfermagem, 53, 1-9. doi: 10.1590/s1980-220x2018008103450

TABORDA, A. L. C. G. (2015). Aspectos da resistência do aluno de medicina na busca por auxílio psicológico [Tese de Doutorado, Faculdade de Medicina da Universidade de São Paulo].

World Health Organization. (2017). Depression and other common mental disorders: Global Health Estimates. https://apps.who.int/iris/bitstream/ handle/10665/254610/WHO-MSD-MER2017.2-eng.f;jsessionid $=451969171794$ C84AE2 23B6434EE1E617? sequence $=1$

World Health Organization. (2018). Mental health: strengthening our response. https://www.who. int/en/news-room/fact-sheets/detail/mentalhealth-strengthening-our-response. 